\title{
Resuscitative Endovascular Balloon Occlusion of the Aorta区REBOA区 in A Swine Model of Iliac Artery Hemorrhage under the Guidance of Portable Ultrasound
}

\author{
Yuqing Huang \\ Medical School of Chinese PLA \\ Xuexia Shan \\ Hainan Hospital of PLA General Hospital \\ Shengzheng Wu \\ Hainan Hospital of PLA General Hospital \\ Xianghui Chen \\ Medical Shool of Chinese PLA \\ Xingxi Lin \\ Hainan Hospital of PLA General Hospital
}

\section{Liye Zhang}

Hainan Hospital of PLA General Hospital

Zhihui Li

Tsinghua University

Faqin Lv ( $\square$ Ivjin8912@163.com )

Medical School of Chinese PLA

\section{Research article}

Keywords: Iliac Artery, Hemorrhage, REBOA, Portable Ultrasound, Animal experiment

Posted Date: March 22nd, 2021

DOI: https://doi.org/10.21203/rs.3.rs-280129/v1

License: (c) (i) This work is licensed under a Creative Commons Attribution 4.0 International License. Read Full License 


\section{Abstract}

Background: The major challenge of applying Resuscitative Endovascular Balloon Occlusion of the Aorta (REBOA) in the pre-hospital setting is to accurately place the balloon in. To evaluate the effects of applying portable ultrasound to guide REBOA for iliac artery hemostasis.

Methods: We first established a swine model of hemorrhage by percutaneously puncture the right iliac artery under the guidance of portable ultrasound. Then we randomly divided the swine into two groups. We recorded systolic pressure $(\mathrm{SP})$, diastolic pressure (DP), heart rate (HR), and the maximum depth of the ascites at baseline (T1), free bleeding for the 30 s (T2), bleeding for $10 \mathrm{~min}$ (T3), and bleeding for 30min (T4). Immediately after T2, we performed REBOA under the guidance of portable ultrasound in the intervention group and manual extracorporeal compression by dry gauze in the control group. We collected total blood loss at T4.

Results: There were 11 swine included in the analysis (intervention group=6, control group=5). The characteristics of the two groups were similar at T1. After punctured the right iliac artery, hemorrhagic shock appeared in both groups at T2 - BP and DP fell, HR elevated, and the maximum depth of the ascites increased. After performing REBOA, SP(in $\mathrm{mmHg}$ ) in the intervention group significantly increased to $97.17 \pm 11.92$ at $\mathrm{T} 3$ and remained stable throughout $\mathrm{T} 4$; while SP in the control group kept decreasing and reached $62.40 \pm 3.44$ at T4. A similar trend was found in DP. HR(in $\mathrm{bpm}$ ) in the intervention group increased from $101.50 \pm 5.39$ in T2 to $111.83 \pm 7.39$ in T3 and stabilized at $113.83 \pm 5.49$ in T4; in the control group, it kept increasing from $103.20 \pm 3.70$ in T2 to $132.40 \pm 3.98$ in T4. The maximum depth of the ascites increased between T2 and T4 in both groups, but significantly slower in the intervention group (at T4 $3.50 \pm 0.36 \mathrm{~cm}$ vs $5.14 \pm 0.35 \mathrm{~cm}, \mathrm{P}<0.05)$. The total blood loss was significantly less in the intervention group $(1245.23 \pm 190.07 \mathrm{~g})$ than in the control group $(2605.63 \pm 291.67 \mathrm{~g})$.

Conclusions: Performing REBOA under the guidance of portable ultrasound can improve the effectiveness of iliac artery hemostasis and have great potential to save lives in pre-hospital settings.

\section{Introduction}

Junctional hemorrhage belongs to non-compressible torso hemorrhage (NCTH), which is defined as hemorrhage that occurs at the junction of an extremity with the torso of the body at an anatomic location, including the base of the neck $^{[1]}$. There are areas where preclude the effectiveness of using an extremity tourniquet to control the bleeding, which contributes to a high risk of death from junctional hemorrhage ${ }^{[2]}$. A study on U.S. combat fatalities showed that between 2001 and 2011, 17.5\% (171/976) of the preventable prehospital combat deaths were caused by junctional hemorrhage $^{[3]}$. Another study showed a mortality rate of $45 \%$ among the patients with junctional hemorrhage at prehospital times less than 30 minutes and remained substantial throughout subsequent prehospital time intervals ${ }^{[4]}$.

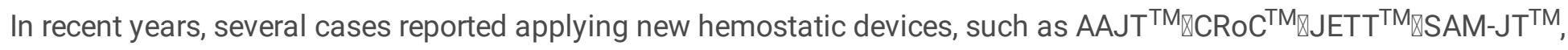
among patients with junctional hemorrhage in the prehospital settings ${ }^{[5-8]}$. However, the effectiveness was only at $24 \rrbracket$ $48 \%{ }^{[9]}$ in the pre-hospital and the transport process. Resuscitative Endovascular Balloon Occlusion of the Aorta (REBOA) is an emerging strategy for junctional hemostasis and has been applied in the pre-hospital settings. In a retrospective study on trauma management, it showed that using REBOA was significantly associated with lower mortality (adjusted odds ratio of survival, 7.430; $95 \% \mathrm{Cl}, 1.081-51.062)^{[10]}$. However, the usage of REBOA was largely limited by the ability to accurately place the balloon in. In the current application, REBOA is usually carried out in the pre-hospital setting without the aid of imaging equipment or guided by X-rays. A study showed that six of the 19 
REBOA attempts (32\%) in trauma patients with exsanguinating pelvic hemorrhage were unsuccessful in the prehospital setting, mainly due to the inaccuracy to locate the bleeding sites or to place the ballon in ${ }^{[11]}$.

Portable ultrasound is currently the only visual instrument that can be applied in the prehospital setting of trauma. With visual ultrasound images, pre-hospital rescuers would be able to accurately locate the bleeding sites and place the ballon at the proximal end of the injured vessel under the portable ultrasound guidance. However, the effectiveness of applying portable ultrasound in REBOA in the pre-hospital setting has not been assessed. In this study, we will establish a swine model of iliac artery hemorrhage with portable ultrasound - we will a 16-gauge core needle biopsy to percutaneously puncture the right iliac artery, which is expected to provide an ideal animal model for hemostasis study at the junction. After establishing the model, we applied REBOA under the guidance of portable ultrasound in the intervention group and manual extracorporeal compression by dry gauze in the control group. We compared a series of indicators, including systolic pressure, diastolic pressure, heart rate, the maximum depth of the ascites, total blood loss, etc., between the two groups.

\section{Materials And Methods}

\subsection{Experimental apparatus and instrument}

The portable ultrasound equipment we used in this study was PHILIPS CX50 portable Color Doppler Ultrasonic Diagnosis Apparatus (Philips Healthcare, Andover, MA, USA), which has an L9-3 linear probe with a frequency of 7-12 $\mathrm{MHz}$. We monitored invasive blood pressure through the TranStarR Single Monitoring Kit (Smiths Med ical ASD, Inc. Dublln, USA) and TranStarR Pressure Monitoring System. To establish the hemorrhage model, all swine underwent core needle biopsy (Bard Biopsy Systems, Franklin Lakes, NJ, USA; type: MC1616, gauge size: $16 \mathrm{~g}$, length of sample notch: $1.9 \mathrm{~cm}$ ) (Fig. 1). The balloon was the Armada 35 / Armada 35 LL PTA Catheter (Abbott Molecular, De Plaines, IL, USA) (Fig. 2).

\subsection{Animal preparation}

Fig. 3 shows the timeline of the study schema. Twelve Wuzhishan swine were provided by the Institute of Animal Science, Hainan provincial Academy of Agricultural Sciences. All the animals were male, with an average body mass of $14.0 \pm 1.1 \mathrm{~kg}$, ranging from 12.5 to $16.0 \mathrm{~kg}$. They adapted to the experimental environment for 3 days and fasted for 12 hours before the experiment. The procedures for the care and use of animals were approved by the Ethics Committee of the PLA general hospital. All applicable institutional and governmental regulations concerning the ethical use of animals were followed.

A mixture of the Zoletil ${ }^{\circledR} 50$ (Virbac, Carros, France) and Sumianxin $\otimes$ (Military Veterinary Institute, China) in a ratio of $1: 2$ was injected intramuscularly into the hind leg of the swine at $0.1 \mathrm{ml} / \mathrm{kg}$ body mass. We placed the animals in the supine position and attached their limbs to the laboratory bench. The hairs were removed from the cervical region, belly, and groin of the swine. Under the guidance of portable ultrasound, we inserted a central venous catheter percutaneously into the right internal jugular vein for follow-up anesthesia. Sequential intravenous administration of $1 / 3$ of the initial dose was given according to the animal's conscious state. The anesthesia was successful with blood pressure, respiration, and heart rate stable and the shallow sensation of the animal disappear. To simulate a severe pre-hospital setting, fluid resuscitation was limited. We cannulated the left common carotid artery with a catheter under the guidance of ultrasound for arterial pressure monitoring and then connected with TranStarR Single Monitoring Kit. Continuous monitoring and recording of vital signs were achieved through the TranStarR Pressure Monitoring System. 


\subsection{Experimental procedure}

We wrapped the ultrasonic probe in sterile plastic wrap. After applying an appropriate amount of acoustic coupling agent to the abdomen and both sides of the inguinal area of the swine, we selected a high-frequency linear probe for an ultrasound examination. Before established the swine bleeding model from the iliac artery, we measured the internal diameters of the abdominal aorta, and both iliac arteries as well as the ascites which was represented by the maximum depth of anechoic zone by using portable ultrasound. We observed the morphology and hemodynamic changes of the iliac artery via two-dimensional ultrasound and color Doppler ultrasound.

We selected the right iliac artery as the injury target. To establish the bleeding model, the appropriate puncture route was determined under portable ultrasound guidance and the puncture procedure was done by using freehand ultrasound guidance. The needle tip of the disposable puncture biopsy device was advanced under ultrasound guidance until it was positioned in the anterior lateral wall of the iliac artery after passing through the skin and muscle layer. Then we initiated the trigger to puncture the iliac artery with a 60-80 angle(video1). We observed the internal diameter and hemodynamics of the iliac artery by ultrasound. A period of free bleed lasting for 30 seconds should be maintained after puncturing the iliac artery ${ }^{[12]}$. According to the manifestations of arterial hemorrhage, a simple standard of the successful model was as follows. We observed a decrease in blood pressure, as well as tachycardia. Two-dimensional ultrasound showed that the iliac artery collapsed with a narrowed internal diameter and the intraperitoneal anechoic zone was gradually expanding.

After the completion of animal models, they were randomly divided into two groups $(n=6)$ in all the models. In the intervention group, we performed REBOA by portable ultrasound guidance to achieve aortic occlusion completely. Under ultrasound guidance, the balloon was placed at the lower abdominal aorta via the Seldinger technique and by inflating the balloon catheter to 4ATM pressure. (Fig. 4). Once the balloon was inflated, the Doppler spectra confirmed a total occlusion of the arterial. In the control group, we performed extracorporeal compression by gauze locally. Especially, the operator was the same person, and the similar degree of force the operator uses to press the gauze.

\subsection{Data collection}

We collected the physiologic parameters in real-time by using the TranStarR Pressure Monitoring System. Parameters measured included systolic pressure (SP), diastolic pressure (DP), and heart rate (HR). And we obtained the maximum depth of anechoic zone in the abdominal cavity by ultrasound at baseline (T1), free bleeding for the 30s (T2), bleeding for 10min (T3), bleeding for 30min (T4). To confirm the effect of gross pathology, the swine were sacrificed by deep anesthesia and air embolism after T4. We opened the abdominal and retroperitoneum to identify the rupture and bleeding of the iliac artery. The bleeding mass was measured by the weight of the hemostatic gauze. We took the muscle tissue in the same part of the right lower limb for pathological samples by using a 16-gauge core needle biopsy in T1 and T4. These tissues were soaked in 10\% normal buffered formalin immediately for one week, and then dehydrated with ethanol and embedded in paraffin. The 4- $\mu \mathrm{m}$-thick muscle sections were stained with $\mathrm{HE}$ and the pathological changes of muscle tissue were observed under the light microscope.

\subsection{Statistical Analysis}

We assessed all data for normality and presented as means \pm standard error of the mean. Statistical analysis was performed using SPSS19.0 (SPSS, Chicago, IL, USA) and graphs were generated using the GraphPad Prism 8 (GraphPad Software, San Diego, CA, USA). P < 0.05 was considered statistically significant. The homogeneity of the baseline values of swine and the total blood loss between the two groups were checked via independent t-tests. Besides, to determine the statistical significance of differences in a series of indicators (SP, DP, HR, maximum depth of 
anechoic zone) between REBOA and gauze, we adopted a repeated-measures ANOVA (RMANOVA) for analysis, with time as the within-group factor and treatment as the between-group factor. And to protect against Type I errors of the RMANOVA, the degrees of freedom were corrected using the Greenhouse-Geisser method when sphericity assumptions were not upheld. If there was a significant group*time interaction, indicating that the groups in the analysis demonstrated significantly different time courses, post-hoc analyses were performed using one-way ANOVAs at each time point in the within-group.

\section{Results}

\subsection{Baseline Data}

We recruited 12 swine for this study while one swine in the control groups was excluded because major bleeding occurred during puncture due to carotid artery tortuosity. Eventually, eleven swine were included in the final sample. All the general physiological conditions were statistically similar between the two groups in T1, including weight $(14.08 \pm 1.28 \mathrm{~kg}$ VS $14.10 \pm 0.10 \mathrm{~kg}, P=0.541)$, SP $(121.17 \pm 11.51 \mathrm{mmHg}$ VS $118.40 \pm 12.34 \mathrm{mmHg}, P=0.710)$, DP $(91.50 \pm 12.05 \mathrm{mmHg}$ VS $87.80 \pm 15.55 \mathrm{mmHg}, P=0.712)$, HR (80.83 $\pm 6.11 \mathrm{bpm}$ VS $82.40 \pm 4.28 \mathrm{bpm}, P=0.642)$, abdominal aortic diameter $(0.69 \pm 0.05 \mathrm{~cm}$ VS $0.65 \pm 0.06 \mathrm{~cm}, P=0.475)$, and the maximum depth of the anechoic zone in the abdominal cavity $(0.07 \pm 0.08 \mathrm{~cm}$ VS $0.12 \pm 0.13 \mathrm{~cm}, P=0.230)$. Detailed statistics are reported in Table 1 .

Table 1. Sample characteristics at baseline (Mean \pm SD).

\begin{tabular}{|llllllll|}
\hline Group & Number & $\begin{array}{l}\text { Weight } \\
(\mathrm{kg})\end{array}$ & $\begin{array}{l}\text { Abdominal } \\
\text { Aortic } \\
\text { Diameter } \\
(\mathrm{cm})\end{array}$ & $\begin{array}{l}\text { Systolic } \\
\text { Pressure } \\
(\mathrm{mmHg})\end{array}$ & $\begin{array}{l}\text { Diastolic } \\
\text { Pressure } \\
(\mathrm{mmHg})\end{array}$ & $\begin{array}{l}\text { Heart } \\
\text { Rate } \\
(\mathrm{bpm})\end{array}$ & $\begin{array}{l}\text { Maximum } \\
\text { Depth of } \\
\text { Anechoic } \\
\text { Zone } \\
(\mathrm{cm})\end{array}$ \\
$\begin{array}{l}\text { Intervention } \\
\text { group }\end{array}$ & 6 & $14.08 \pm 1.28$ & $0.69 \pm 0.05$ & $121.17 \pm 11.51$ & $91.50 \pm 12.05$ & $80.83 \pm 6.11$ & $0.07 \pm 0.08$ \\
$\begin{array}{l}\text { Control } \\
\text { group }\end{array}$ & 5 & $14.10 \pm 0.10$ & $0.65 \pm 0.06$ & $118.40 \pm 12.34$ & $87.80 \pm 15.55$ & $82.40 \pm 4.28$ & $0.12 \pm 0.13$ \\
\hline $\begin{array}{l}T \text { values } \\
P \text { values }\end{array}$ & 0.024 & 1.144 & 0.384 & 0.381 & 0.481 & 0.830 \\
\hline
\end{tabular}

\subsection{Analyzing the factors in the intervention group}

During the process to establish the model, hemorrhagic shock appeared in the intervention group after puncturing the right iliac artery. Including BP fell from $121.17 \pm 11.51 \mathrm{mmHg}$ at T1 to $83.00 \pm 16.64 \mathrm{mmHg}$ at T2 $(P=0.000)$, DP fell from $91.50 \pm 12.05 \mathrm{mmHg}$ at T1 to $60.17 \pm 16.76 \mathrm{mmHg}$ at T2 $(P=0.000), \mathrm{HR}$ increased from $80.83 \pm 6.11 \mathrm{bpm}$ at T1 to $101.50 \pm 5.39 \mathrm{bpm}$ at T2 $(P=0.000)$, and the maximum depth of anechoic zone in the abdominal cavity increased from $0.07 \pm 0.09 \mathrm{~cm}$ at T1 to $2.32 \pm 0.25 \mathrm{~cm}$ at T2 $(P=0.000)$. After measurement was done at T2, we performed REBOA under portable ultrasound guidance. SP and DP increased to $97.17 \pm 11.92 \mathrm{mmHg}(\mathrm{T} 2 \mathrm{vs} \mathrm{T3}, P=0.040)$ and $66.67 \pm 12.34 \mathrm{mmHg}$ ( T2 vs T3, $P=0.214$ ) at T3 respectively. HR and the maximum depth of the anechoic zone increased to $111.83 \pm 7.39 \mathrm{bpm}$ (T2 vs T3, $P=0.001$ ) and $3.10 \pm 0.41 \mathrm{~cm}(\mathrm{~T} 2 \mathrm{vs} T 3, P=0.001$ ) at T3. Then, SP, DP, and HR became stable in T4 at $97.50 \pm 9.29 \mathrm{mmHg}$ (T3 vs T4, $P=0.846), 67.67 \pm 10.78 \mathrm{mmHg}$ (T3 vs T4, $P=0.518$ ), and $113.83 \pm 5.49 \mathrm{bpm}$ (T3 vs T4, $P=0.352$ ), respectively. Yet the maximum depth of the anechoic zone in the abdominal cavity slightly increased to $3.50 \pm 0.36 \mathrm{~cm}$ at T4 (T3 vs T4, $P=0.000$ ). Detailed statistics are reported in Tables 2 and 3. 


\subsection{Analyzing the factors in the control group}

Hemorrhagic shock also appeared in the control group after establishing the model. BP fell from $118.40 \pm 12.34 \mathrm{mmHg}$ at T1 to $85.40 \pm 13.65 \mathrm{mmHg}$ at T2 $(P=0.000)$, DP fell from $87.80 \pm 15.55 \mathrm{mmHg}$ at T1 to $56.20 \pm 7.40 \mathrm{mmHg}$ at T2 ( $P$ $=0.004)$, HR increased from $82.40 \pm 4.28 \mathrm{bpm}$ at T1 to $103.20 \pm 3.70 \mathrm{bpm}$ at T2 $(P=0.000)$, and the maximum depth of anechoic zone in the abdominal cavity increased from $0.12 \pm 0.13 \mathrm{~cm}$ at $\mathrm{T} 1$ to $2.46 \pm 0.25 \mathrm{~cm}$ at T2 $(P=0.000)$. After performing extracorporeal compression by gauze locally, SP kept falling to $71.20 \pm 6.18 \mathrm{mmHg}$ at T3 (T2 vs T3, $P$ $=0.016$ ), and then to $62.40 \pm 3.44 \mathrm{mmHg}$ at T4 (T3 vs T4, $P=0.003$ ). Similarly, DP decreased to $43.80 \pm 5.63 \mathrm{mmHg}$ at T3 (T2 vs T3, $P=0.000$ ), and further to $35.00 \pm 3.39 \mathrm{mmHg}$ at T4 (T3 vs T4, $P=0.001$ ). HR increased significantly to $122.00 \pm 4.53 \mathrm{bpm}$ at T3 (T2 vs T3, $P=0.000$ ) and then to $132.40 \pm 3.98 \mathrm{bpm}$ at T4 (T3 vs $\mathrm{T} 4, P=0.000$ ). The maximum depth of the anechoic zone kept increasing to $4.12 \pm 0.24 \mathrm{~cm}$ at T3 (T2 vs T3, $P=0.000$ ) and further to $5.14 \pm 0.35 \mathrm{~cm}$ at T4 (T3 vs T4, $P=0.000$ ). Detailed statistics are reported in Tables 2 and 3.

\subsection{Analyzing the factors between groups}

The RMANOVA of SP revealed a main effect of group $(F=5.732, P=0.040)$, a main effect of test time $(F=145.103, P$ $=0.000)$, and a significant group * test time interaction $(F=34.081, P=0.000)$. For $D P$, it indicated a main effect of group $(F=6.547, P=0.031)$, a main effect of test time $(F=89.259, P=0.000)$, and a significant group * test time interaction $(F=16.121, P=0.000)$. For $\mathrm{HR}$, it indicated a main effect of group $(F=7.419, P=0.023)$, a main effect of test time $(F=549.658, P=0.000)$, and a significant group * test time interaction $(F=26.597, P=0.000)$. And for the maximum depth of the anechoic zone, it also indicated a main effect of group $(F=27.742, P=0.000)$, a main effect of test time $(F=976.212, P=0.000)$, and a significant group * test time interaction $(F=40.568, P=0.000)$. Detailed statistics are reported in Tables 2 and Fig. 5.

Table 2. The changes in hemodynamic and physiological parameters in the intervention group and control group at different times. 


\begin{tabular}{|c|c|c|c|c|}
\hline Group/time & $\begin{array}{l}\text { Systolic } \\
\text { Pressure } \\
(\mathrm{mmHg})\end{array}$ & $\begin{array}{l}\text { Diastolic } \\
\text { Pressure } \\
(\mathrm{mmHg})\end{array}$ & $\begin{array}{l}\text { Heart } \\
\text { Rate } \\
\text { (bpm) }\end{array}$ & $\begin{array}{l}\text { Maximum Depth of } \\
\text { Anechoic Zone } \quad(\mathrm{cm})\end{array}$ \\
\hline \multicolumn{5}{|l|}{ Intervention group } \\
\hline T1 & $121.17 \pm 11.51$ & $91.50 \pm 12.05$ & $80.83 \pm 6.11$ & $0.07 \pm 0.09$ \\
\hline T2 & $83.00 \pm 16.64$ & $60.17 \pm 16.76$ & $101.50 \pm 5.39$ & $2.32 \pm 0.25$ \\
\hline T3 & $97.17 \pm 11.92$ & $66.67 \pm 12.34$ & $111.83 \pm 7.39$ & $3.10 \pm 0.41$ \\
\hline T4 & $97.50 \pm 9.29$ & $67.67 \pm 10.78$ & $113.83 \pm 5.49$ & $3.50 \pm 0.36$ \\
\hline \multicolumn{5}{|l|}{ Control group } \\
\hline T1 & $118.40 \pm 12.34$ & $87.80 \pm 15.55$ & $82.40 \pm 4.28$ & $0.12 \pm 0.13$ \\
\hline T2 & $85.40 \pm 13.65$ & $56.20 \pm 7.40$ & $103.20 \pm 3.70$ & $2.46 \pm 0.25$ \\
\hline T3 & $71.20 \pm 6.18$ & $43.80 \pm 5.63$ & $122.00 \pm 4.53$ & $4.12 \pm 0.24$ \\
\hline T4 & $62.40 \pm 3.44$ & $35.00 \pm 3.39$ & $132.40 \pm 3.98$ & $5.14 \pm 0.35$ \\
\hline \multirow{2}{*}{$\begin{array}{l}\text { Time }(P \text { values }) \\
{[\text { Time }(F \text { values })]}\end{array}$} & $0.000 *$ & \multirow{2}{*}{$\begin{array}{l}0.000 * \\
{[89.259]}\end{array}$} & $0.000 *$ & $0.000 *$ \\
\hline & [145.103] & & [549.658] & [976.212] \\
\hline \multirow{2}{*}{$\begin{array}{l}\text { Group ( } P \text { values }) \\
\text { [Group ( } F \text { values })]\end{array}$} & $0.040^{*}$ & $0.031 *$ & $0.023^{*}$ & $0.000^{*}$ \\
\hline & [5.732] & [6.547] & [7.419] & [27.742] \\
\hline Time * Group ( $P$ values) & $0.000 *$ & $0.000 *$ & $0.000 *$ & $0.000 *$ \\
\hline [Time * Group ( $F$ values)] & [34.081] & {$[16.121]$} & [26.597] & [40.568] \\
\hline
\end{tabular}

The asterisk $\left(^{\star}\right)$ represents significant differences at $p<0.05$.

T1: at baseline, T2: free bleeding for the $30 \mathrm{~s}, \mathrm{~T} 3$ : bleeding for $10 \mathrm{~min}, \mathrm{~T} 4$ : bleeding for $30 \mathrm{~min}$.

Table 3. Compared with the previous time point in the within-group by using one-way ANOVAs. 


\begin{tabular}{|c|c|c|c|c|}
\hline $\begin{array}{l}\text { Group/ } P \\
\text { values }\end{array}$ & $\begin{array}{l}\text { Systolic Pressure } \\
(\mathrm{mmHg})\end{array}$ & $\begin{array}{l}\text { Diastolic Pressure } \\
(\mathrm{mmHg})\end{array}$ & $\begin{array}{l}\text { Heart Rate } \\
\text { (bpm) }\end{array}$ & $\begin{array}{l}\text { Maximum Depth of Anechoic } \\
\text { Zone }(\mathrm{cm})\end{array}$ \\
\hline \multicolumn{5}{|l|}{$\begin{array}{l}\text { Intervention } \\
\text { group }\end{array}$} \\
\hline$P($ T1 vs T2) & $0.000 *$ & $0.000 *$ & $0.000 *$ & $0.000 *$ \\
\hline$P($ T2 vs T3) & $0.040 *$ & 0.214 & $0.001 *$ & $0.001^{*}$ \\
\hline$P(\mathrm{~T} 3$ vs T4) & 0.846 & 0.518 & 0.352 & $0.000 *$ \\
\hline \multicolumn{5}{|l|}{$\begin{array}{l}\text { Control } \\
\text { group }\end{array}$} \\
\hline$P(\mathrm{~T} 1 \mathrm{vs} \mathrm{T} 2)$ & $0.000 *$ & $0.004^{\star}$ & $0.000 *$ & $0.000 *$ \\
\hline$P($ T2 vs T3) & $0.016 *$ & $0.000 *$ & $0.000 *$ & $0.000 *$ \\
\hline$P($ T3 vs T4) & $0.003^{*}$ & $0.001 *$ & $0.000 *$ & $0.001 *$ \\
\hline
\end{tabular}

The asterisk $(*)$ represents significant differences at $p<0.05$.

T1: at baseline, T2: free bleeding for the 30s, T3: bleeding for $10 \mathrm{~min}, \mathrm{~T} 4$ : bleeding for $30 \mathrm{~min}$.

\subsection{Ultrasound Manifestation}

During the process to establish the model, the artery rapidly collapsed with less blood flow and the inner diameter of the artery decreased in the ultrasound image after the puncture of the iliac artery. Color Doppler ultrasound showed that the iliac vein was compressed and there was no blood flow signal (Fig. 6).

In the intervention group, we placed the balloon catheter from the left iliac artery to the lower abdominal aorta under

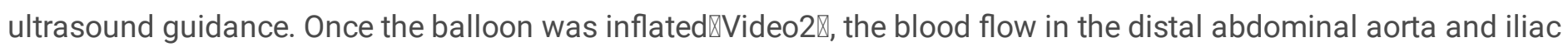
artery were significantly reduced or disappeared by color Doppler ultrasound. (Video3, Fig. 7). Via the Doppler spectrum, we verified that the aorta had occluded with the balloon completely. As the balloon inflated, the ultrasound images clearly showed that the maximum depth of the anechoic zone in the abdominal cavity increased slower in the intervention group than in the control group (Fig. 8).

\subsection{Pathologic finding}

After the animals were sacrificed and the abdominal was opened, huge blood clots in the abdominal and retroperitoneum were visible. The gross pathological finding confirmed the rupture of the right iliac artery (Fig. 9). We measured total blood loss by quantifying blood clots and blood absorbed by the gauze. The total blood loss of the intervention group and the control group was $1245.23 \pm 190.07 \mathrm{~g}$ and $2605.63 \pm 291.67 \mathrm{~g}$ respectively. The difference between the two groups was statistically significant $(T=6.768, P=0.002)$. However, on pathologic assessment, there was not a significant difference in the muscle tissue of both the intervention group and the control group in T1 and T4 (Fig. 10).

\section{Discussions}

There are two major contributions to this study. First, we established a swine model of iliac artery hemorrhage using a 16-gauge core needle biopsy to percutaneously puncture the right iliac artery under portable ultrasound guidance. This method was simple and feasible with a high success rate. Second, we demonstrated the effectiveness of using 
portable ultrasound to guide REBOA for iliac artery hemorrhage. Compared with the traditional method of gauze compression, portable ultrasound guidance allows real-time visualization of balloon implantation, improving accuracy and safety. Between T3 and T4, blood pressure and heart rate became stable in the group using portable ultrasound to guide REBOA; while in the group using gauze compression, blood pressure and heart rate kept deteriorating. Although there was still a small increase in the maximum depth of the ascites in the abdominal cavity, it may be related to the simultaneous injury of the iliac vein or surrounding small vessels during the modeling, and the blood flow in the distal iliac artery could still flow out from the incision. REBOA mainly reduces bleeding at the site of iliac artery injury by blocking the flow of the abdominal aorta, which has limitations for veins and distal arteries.

REBOA is a minimally invasive procedure that introduces a balloon occlusion catheter via a percutaneous groin puncture or cutdown of the femoral artery into the aorta to obtain endovascular aortic occlusion ${ }^{[13,14]}$. REBOA has been used clinically for the treatment of abdominal aortic aneurysm and related obstetric diseases ${ }^{[15,16]}$, but now it has been widely used for the hemostatic treatment of bleeding at NCTH. In a retrospective cohort study that examined the association between the treatment of REBOA and mortality in a group of patients with penetrating trauma to the torso, the odds of death in REBOA group was $78 \%$ lower than that in the non-REBOA group (odds ratio [OR] $0.20,95 \% \mathrm{Cl}$ $0.05-0.77)^{[17]}$. But imaging instruments, intra-arterial placement of REBOA is challenging in the pre-hospital settings. Viktor A et al. placed six sedated male sheep into a low capacity Eurocopter AS-350, and every animal underwent blind (left side) and ultrasound-guided (US) (right side) vascular access (VA) to the femoral artery followed by REBOA. Among six blind punctures, one was successful, 2/6 went into the vein, 3/6 completely failed, and switched to USpunctures (making the total number of US-punctures nine). Eight out of nine US punctures were successful ${ }^{[18]}$.

Our study showed the effectiveness of using portable ultrasound to guide REBOA, which was consistent with previous kinds of literature. Brede JR et al. ${ }^{[19]}$ performed pre-hospital resuscitation balloon occlusion of an aortic lumen in 10 patients with non-traumatic cardiac arrest guided by ultrasound on a helicopter. The success rate of the first intubation of REBOA was as high as $80 \%$. With the development of portable ultrasound, it has been such an important imaging instrument in the pre-hospital setting and appeared to have a high sensitivity and specificity. In the study of 756 patients with severe pre-hospital trauma, pulmonary ultrasound in the pre-hospital setting was found to have higher diagnostic accuracy for hemothorax and pneumothorax than chest X-ray, and the sensitivity and specificity of abdominal ultrasound in detecting peritoneal effusion were $70 \%$ and $96 \%$ respectively ${ }^{[20]}$. Portable ultrasound is not only an effective method to examine intra-abdominal traumatic hemorrhage, also can be used to guide timely hemostasis by injecting hemostatic agents. Through animal experiments, Li W et al ${ }^{[21]}$ proved that percutaneous injection of hemocoagulase and a-cyanoacylatee under the guidance of contrast-enhanced ultrasound could achieve immediate hemostasis for severe closed hepatosplenic rupture hemorrhage with remarkable effect. In the medical management of heavy casualties and disasters, the injuries of casualties are classified through pre-hospital ultrasound in hopes of fully utilizing medical resources efficiently, which realize the rational distribution of medical resources and save more people ${ }^{[22]}$. Although using portable ultrasound to guide REBOA for NCTH in the pre-hospital has been widely recognized to have great potentials, there are not many previous studies that have revealed its effects. This is a study showing that portable ultrasound is a feasible and effective method to be combined with REBOA in the pre-hospital setting for iliac artery hemostasis.

This study has several limitations: First, when REBOA is occluded for more than 80 minutes, it can cause severe vascular paralysis, systemic inflammation, and lactic acidosis, leading to potentially fatal ischemia of the trunk, viscera, and spinal cord ${ }^{[11,23]}$. However, in this study the intervention was only implemented within $30 \mathrm{~min}$ after the swine model of iliac artery hemorrhage was established, and there were no significant changes in histopathology. The relationship between occlusion time and adverse complications could be further studied in subsequent experiments.

Page 9/19 
The possibility and efficacy of partial REBOA in the pre-hospital setting will also have to be evaluated in future research ${ }^{[24]}$. Second, although this study limited the amount of fluid resuscitation supplement to simulate the prehospital setting, its hemostatic effect can be further studied in a pre-hospital setting in subsequent experiments. Last, in the process of balloon placement, the time required depends on the operator's experience and technology. The hemostatic effect is also related to the time of balloon placement, so the training of the operator is also essential.

\section{Conclusion}

Compared with gauze compression for hemostasis, REBOA under portable ultrasound guidance can achieve rapid and effective hemostasis in the event of injury and bleeding at the junction. As the only imaging instrument that can be used for diagnosis and treatment in the pre-hospital setting, portable ultrasound could largely improve the accuracy and effectiveness of REBOA in the hemostasis in the pre-hospital junction. Via ultrasound visualization, it can quickly locate the bleeding site of the injury, and also help accurately place the balloon in for hemostasis treatment.

\section{Abbreviations List}

\begin{tabular}{ll} 
REBOA & Resuscitative Endovascular Balloon Occlusion of The Aorta \\
\hline $\mathrm{CH}$ & Compressive Hemorrhage \\
\hline NCTH & Non-Compressive Torso Hemorrhage \\
\hline HE & Hematoxylin and Eosin staining \\
\hline SP & Systolic Pressure \\
\hline DP & Diastolic Pressure \\
\hline HR & Heart Rate
\end{tabular}

\section{Declarations}

Ethics approval: The experiment was approved by the Ethics Committee of the PLA General Hospital.

Consent for publication: Not applicable.

Availability of data and materials: The data that support the findings of this study are available from the corresponding author upon reasonable request.

Competing interests: We have non-financial competing interests.

Funding[This work was supported by The Military Innovation Research Foundation under Grant No.CX19025; and Science and Technology Major Project of Hainan Province under Grant No. ZDKJ2019012; and RESEARCH and Development Program of Hainan Province under Grant No.ZDYF2016132; and Medical and Health Science and Technology Innovation Project of Sanya City under Grant No.2018YW01; and Military Medical Research Program of PLA General Hospital under Grant No. QNC19050.

Authors' contributions $\square$ We certify that we have participated sufficiently in the work. Yuqing Huang and Xuexia Shan contributed to the work equally and should be regarded as co-first authors. Zhihui Li and Faqin Lv contributed to the work equally and should be regarded as co-corresponding authors. Shengzheng Wu, Faqin Lv, and Yuqing Huang take responsibility for the appropriateness of the experimental design and method. Xianghui Chen, Xuexia Shan, Yuqing 
Huang, Xingxi Lin, and Liye Zhang take public responsibility to complete the experiment. And Yuqing Huang, Xuexia Shan, and Zhihui Li take responsibility for the collection, analysis of the data. Yuqing Huang, Xuexia Shan, Zhihui Li, and Faqin Lv wrote and revised this manuscript.

Acknowledgments: Not applicable.

\section{References}

1. Kotwal, Butler, Gross, Kheirabadi, Baer, Dubick, et al. Management of Junctional Hemorrhage in Tactical Combat Casualty Care: TCCC Guidelines?Proposed Change 13-03. Journal of special operations medicine : a peer reviewed journal for SOF medical professionals 2013;13(4):85-93.

2. Kotwal, Butler. Junctional Hemorrhage Control for Tactical Combat Casualty Care. Wilderness Environ Med 2017;28(2S):S33-S38. doi: 10.1016/j.wem.2016.11.007.

3. Eastridge, Mabry, Seguin, Cantrell, Tops, Uribe, et al. Death on the battlefield (2001-2011): implications for the future of combat casualty care. The journal of trauma and acute care surgery 2012;73:S431-437. doi: 10.1097/TA.0b013e3182755dcc.

4. Eastridge, Holcomb, Shackelford. Outcomes of traumatic hemorrhagic shock and the epidemiology of preventable death from injury. Transfusion 2019;59(S2):1423-1428. doi: 10.1111/trf.15161.

5. J. Abdominal aortic and junctional tourniquet controls hemorrhage from a gunshot wound of the left groin. Journal of special operations medicine : a peer reviewed journal for SOF medical professionals 2014;14(2):6-8.

6. Kragh, Johnson, Henkel, Dubick. Technique of axillary use of a Combat Ready Clamp to stop junctional bleeding. Am J Emerg Med 2013;31(8):1274-1276. doi: 10.1016/j.ajem.2013.02.027.

7. KS, L, JB. Prehospital emergency care: evaluation of the junctional emergency tourniquet tool with a perfused cadaver model. Journal of special operations medicine : a peer reviewed journal for SOF medical professionals 2014;14(1):40-44.

8. JK, M, BL, AA, G, AM, et al. First case report of SAM(r) Junctional tourniquet use in Afghanistan to control inguinal hemorrhage on the battlefield. Journal of special operations medicine : a peer reviewed journal for SOF medical professionals 2014;14(2):1-5.

9. Gaspary, Zarow, Barry, Walchak, Conley, Roszko. Comparison of Three Junctional Tourniquets Using a Randomized Trial Design. Prehosp Emerg Care 2019;23(2):187-194. doi: 10.1080/10903127.2018.1484968.

10. Otsuka, Sato, Sakurai, Aoki, Yamagiwa, lizuka, et al. Effect of resuscitative endovascular balloon occlusion of the aorta in hemodynamically unstable patients with multiple severe torso trauma: a retrospective study. World $\mathrm{J}$ Emerg Surg 2018;13:49. doi: 10.1186/s13017-018-0210-5.

11. Lendrum, Perkins, Chana, Marsden, Davenport, Grier, et al. Pre-hospital Resuscitative Endovascular Balloon Occlusion of the Aorta (REBOA) for exsanguinating pelvic haemorrhage. Resuscitation 2019;135:6-13. doi: 10.1016/j.resuscitation.2018.12.018.

12. Sokol, Black, Shawhan, Marko, Eckert, Tran, et al. Efficacy of a novel fluoroscopy-free endovascular balloon device with pressure release capabilities in the setting of uncontrolled junctional hemorrhage. J Trauma Acute Care Surg 2016;80(6):907-914. doi: 10.1097/TA.0000000000001051.

13. JM, JW, A, NP, JR, TE. Endovascular balloon occlusion of the aorta is superior to resuscitative thoracotomy with aortic clamping in a porcine model of hemorrhagic shock. Surgery 2011;150(3):400-409. doi:

10.1016/j.surg.2011.06.010.

Page $11 / 19$ 
14. Manzano-Nunez, Escobar-Vidarte, Orlas, Herrera-Escobar, Galvagno, Melendez, et al. Resuscitative endovascular balloon occlusion of the aorta deployed by acute care surgeons in patients with morbidly adherent placenta: a feasible solution for two lives in peril. World journal of emergency surgery : WJES 2018;13:44. doi: 10.1186/s13017-018-0205-2.

15. AN, CK. Endovascular proximal control of ruptured abdominal aortic aneurysms: the internal aortic clamp. The Journal of cardiovascular surgery 2009;50(3):381-385.

16. CA, R, MW, TE, AJ, JP, et al. Prophylactic use of resuscitative endovascular balloon occlusion of the aorta in women with abnormal placentation: A systematic review, meta-analysis, and case series. The journal of trauma and acute care surgery 2018;84(5):809-818. doi: 10.1097/ta.0000000000001821.

17. García, Manzano-Nunez, Orlas, Ruiz-Yucuma, Londoño, Salazar, et al. Association of resuscitative endovascular balloon occlusion of the aorta (REBOA) and mortality in penetrating trauma patients. European Journal of Trauma and Emergency Surgery 2020. doi: 10.1007/s00068-020-01370-9.

18. Reva, Perevedentcev, Pochtarnik, Khupov, Kalinina, Samokhvalov, et al. Ultrasound-guided versus blind vascular access followed by REBOA on board of a medical helicopter in a hemorrhagic ovine model. Injury 2020. doi: 10.1016/j.injury.2020.09.053.

19. Brede, Lafrenz, Klepstad, Skjaerseth, Nordseth, Sovik, et al. Feasibility of Pre-Hospital Resuscitative Endovascular Balloon Occlusion of the Aorta in Non-Traumatic Out-of-Hospital Cardiac Arrest. J Am Heart Assoc 2019;8(22):e014394. doi: 10.1161/JAHA.119.014394.

20. Bulger, Perina, Qasim, Beldowicz, Brenner, Guyette, et al. Clinical use of resuscitative endovascular balloon occlusion of the aorta (REBOA) in civilian trauma systems in the USA, 2019: a joint statement from the American College of Surgeons Committee on Trauma, the American College of Emergency Physicians, the National Association of Emergency Medical Services Physicians and the National Association of Emergency Medical Technicians. Trauma Surg Acute Care Open 2019;4(1):e000376. doi: 10.1136/tsaco-2019-000376.

21. Li, Tang, Lv, Zhang, Zhang, An. Effectiveness and safety of CEUS-guided haemostatic injection for blunt splenic trauma: an animal experiment. Radiol Med 2010;115(7):1080-1086. doi: 10.1007/s11547-010-0573-5.

22. Lee, Balk, Schafer, Welwarth, Hardin, Yarza, et al. Accuracy of Focused Assessment with Sonography for Trauma (FAST) in Disaster Settings: A Meta-Analysis and Systematic Review. Disaster Med Public Health Prep 2019;13(56):1059-1064. doi: 10.1017/dmp.2019.23.

23. Perkins, Lendrum, Brohi. Resuscitative endovascular balloon occlusion of the aorta: promise, practice, and progress? Curr Opin Crit Care 2016;22(6):563-571. doi: 10.1097/MCC.0000000000000367.

24. Matsumura, Matsumoto, Kondo, Idoguchi, Ishida, Kon, et al. Fewer REBOA complications with smaller devices and partial occlusion: evidence from a multicentre registry in Japan. Emergency medicine journal : EMJ

2017;34(12):793-799. doi: 10.1136/emermed-2016-206383.

\section{Figures}




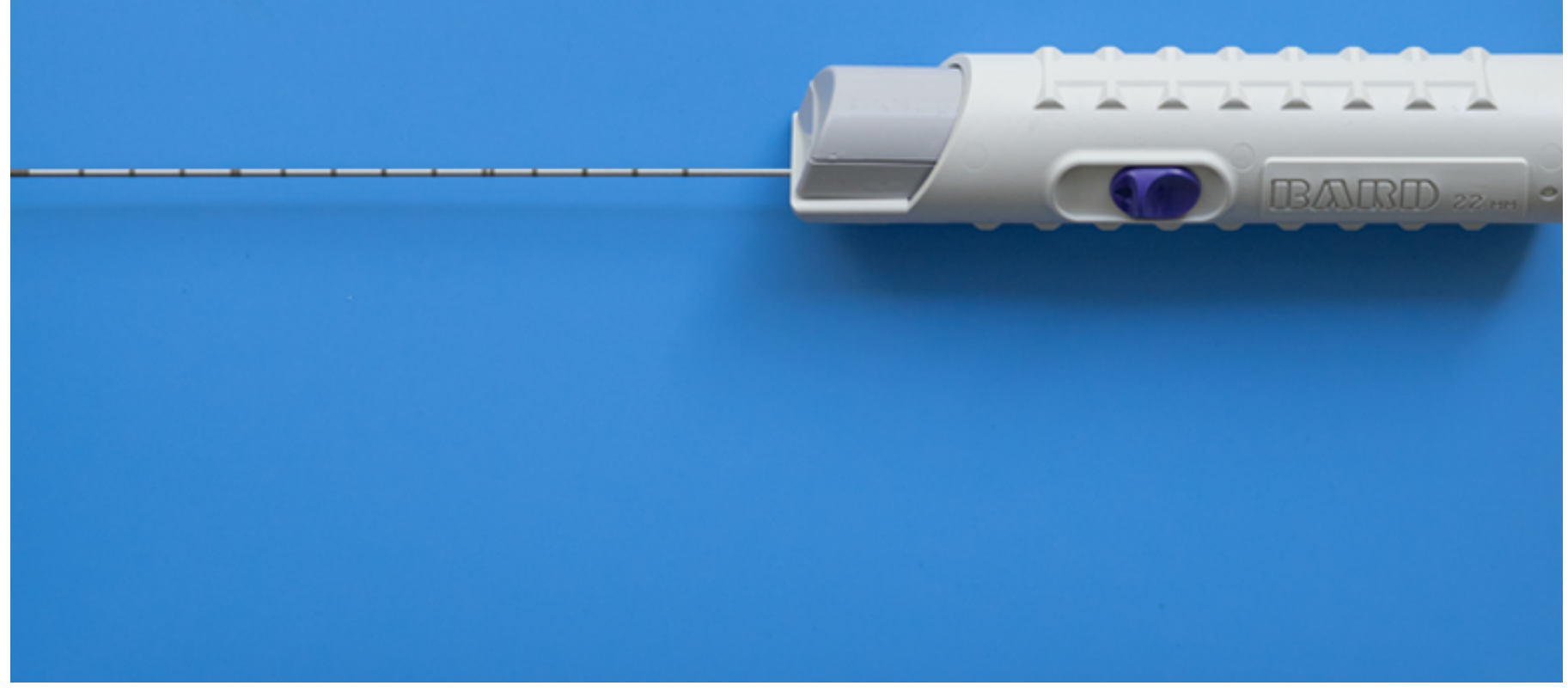

\section{Figure 1}

The core needle biopsy used in establishing the model. Type: MC1616, gauge size: $16 \mathrm{~g}$, length of sample notch: 1.9 $\mathrm{cm}$.
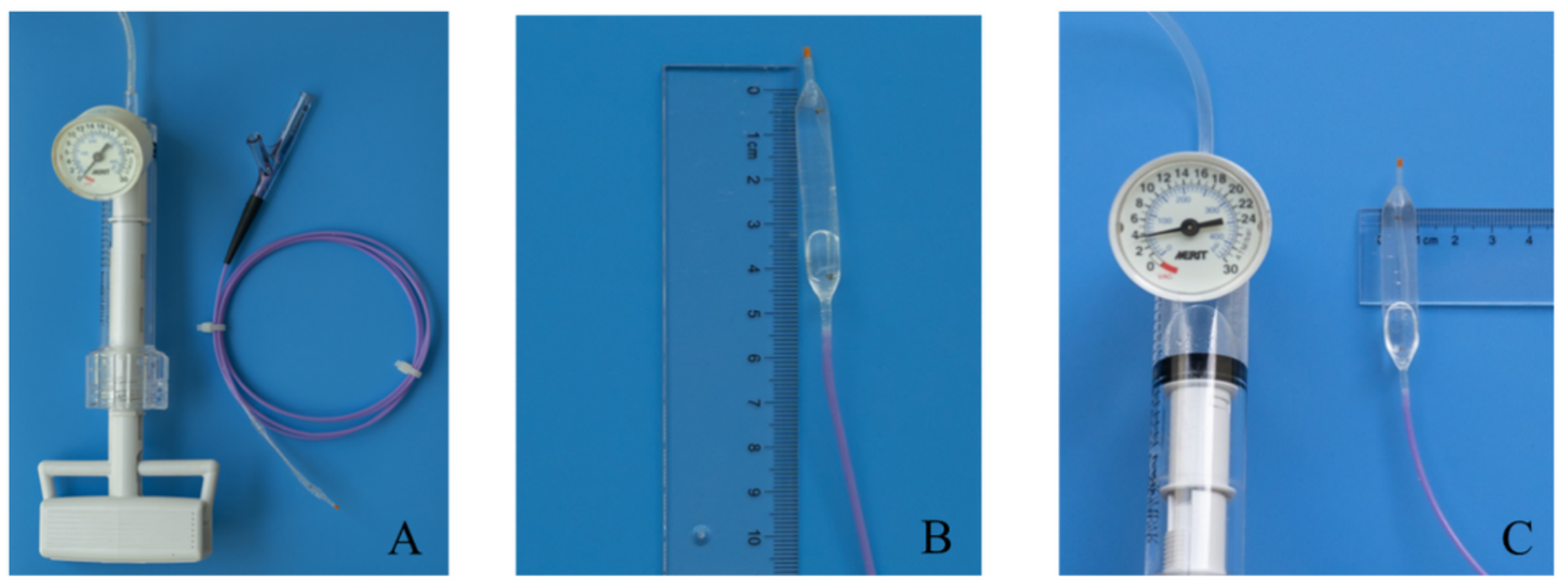

\section{Figure 2}

The balloon used for REBOA. A: The endovascular balloon of aorta occlusion and pressure pump. B: The length of the balloon is about $60 \mathrm{~mm}$; C: When the pressure inside the balloon is 4 ATM (standard atmospheric pressure), the 
diameter of the balloon is $10 \mathrm{~mm}$.

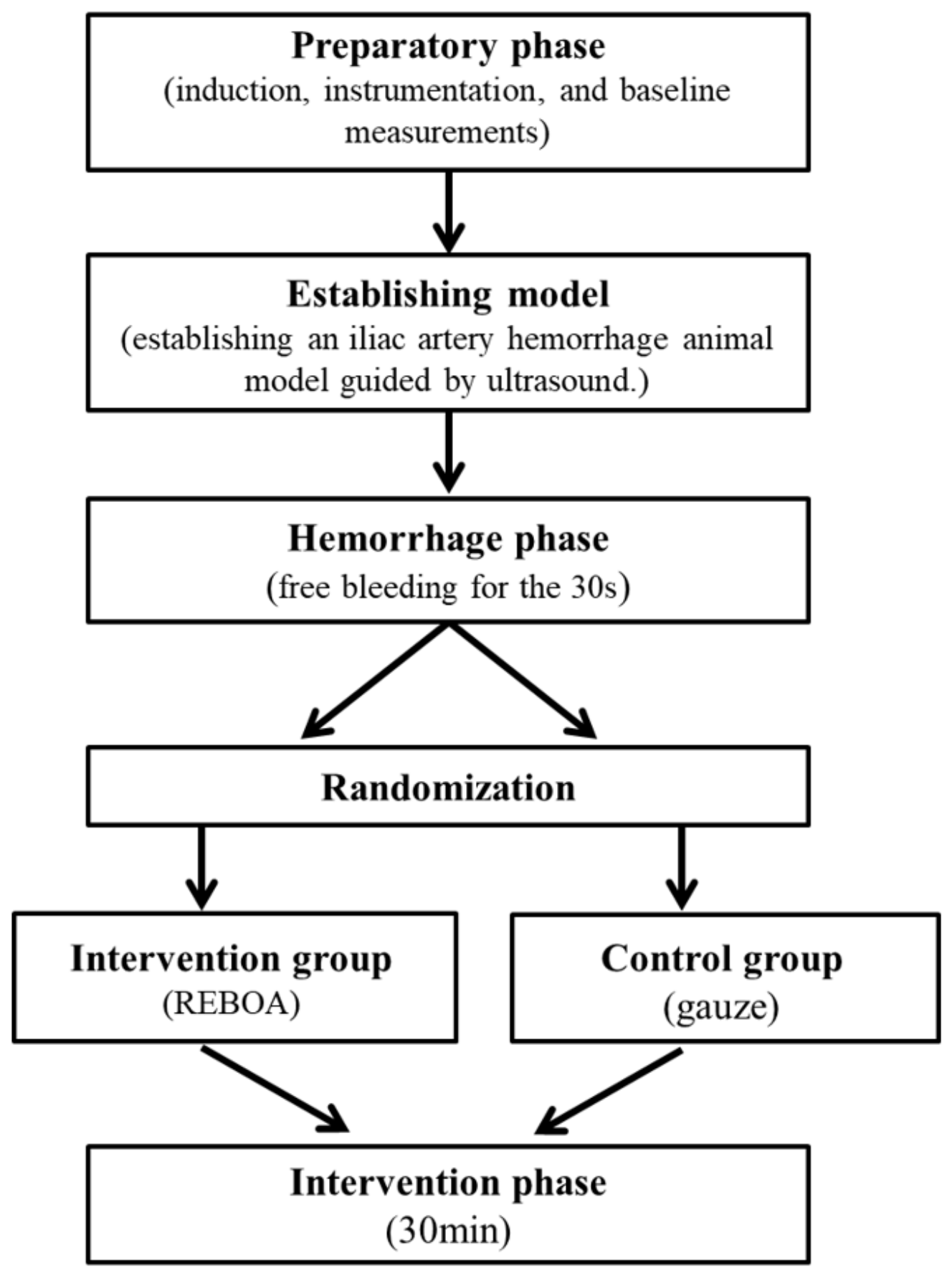

Figure 3

Study schema. 

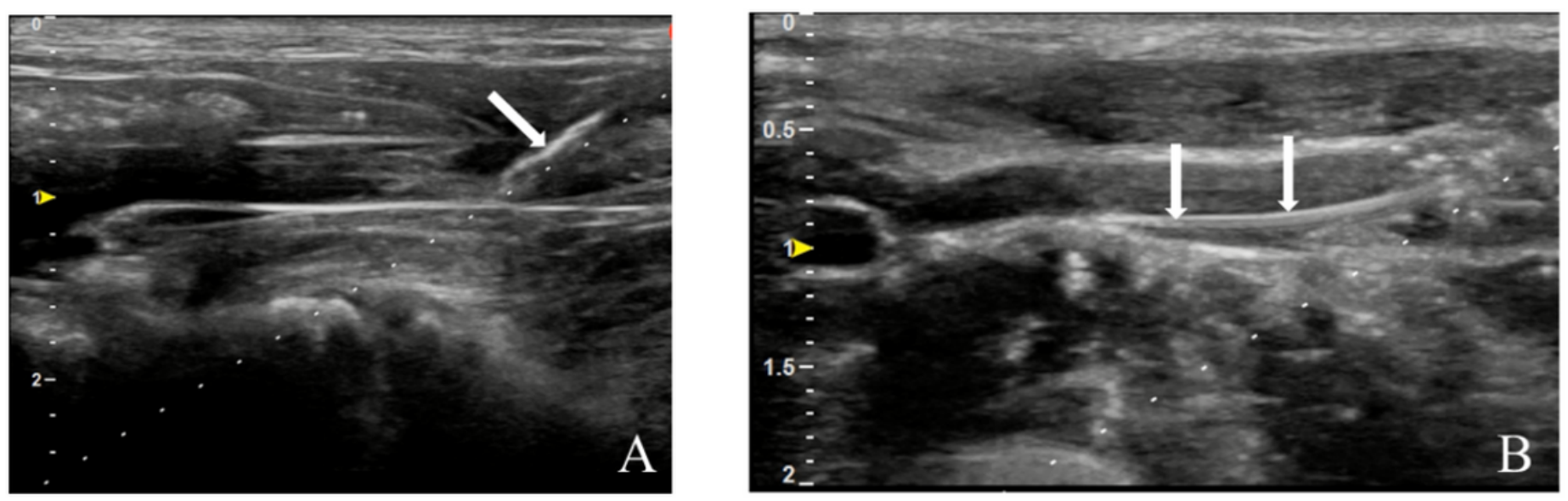

\section{Figure 4}

The iliac artery was punched and the balloon was placed under portable ultrasound guidance. A: Under ultrasound guidance, an endovascular balloon was placed at the lower abdominal aorta via the Seldinger technique. The arrow points to the needle tip. B: The guidewire is carried along the iliac artery. The arrow points to the wire. 

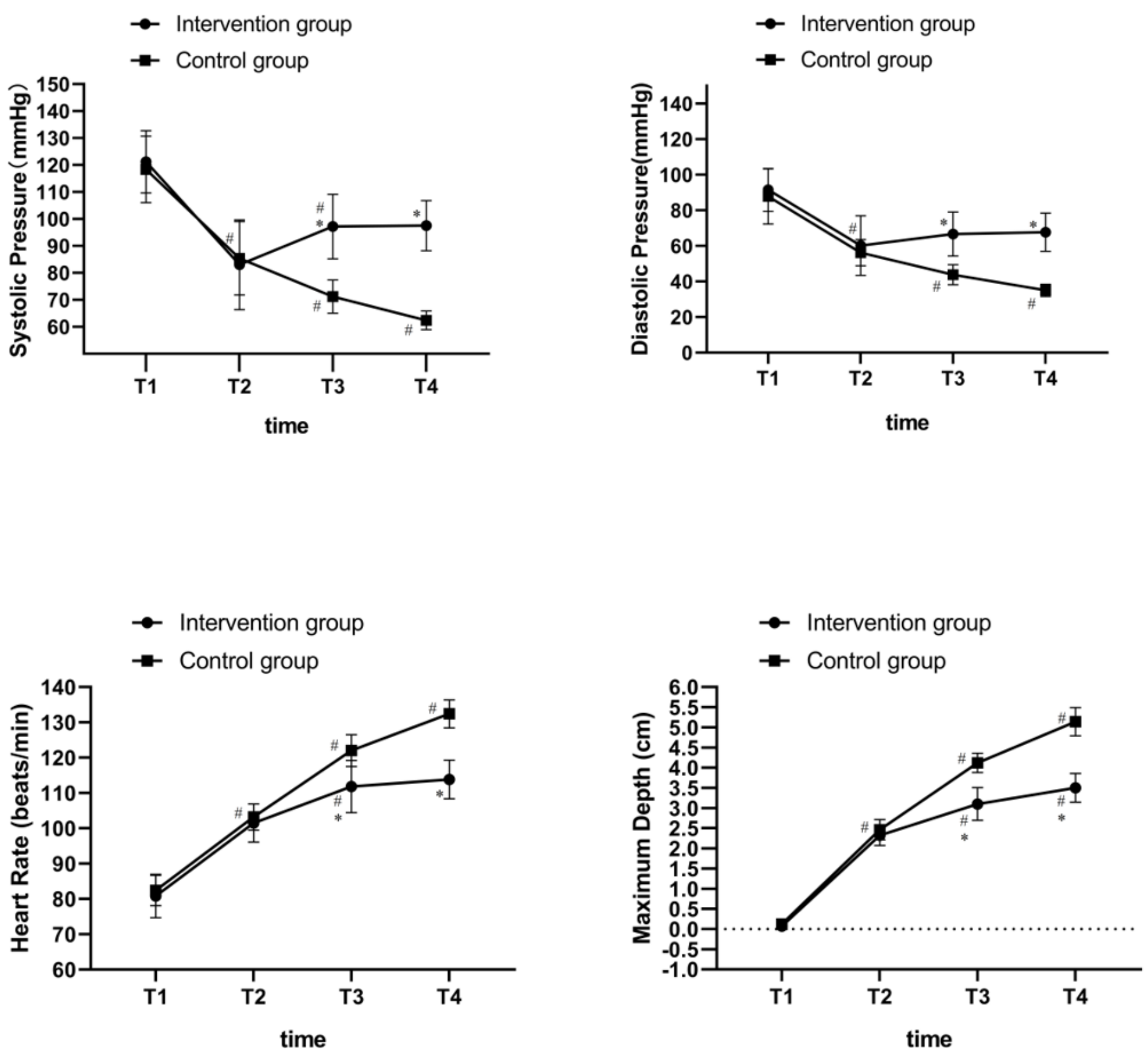

Figure 5

Comparison of SP, DP, HR, and the maximum depth of the anechoic zone over time between two groups. The RMANOVA of SP, DP, HR, and the maximum depth of the anechoic zone revealed a main effect of group ( all $P<0.05$ ), a main effect of test time ( all $P<0.05$ ), and a significant group * test time interaction $($ all $P<0.05)$. Compared with the control group: ${ }^{\star} \mathrm{P}<0.05$. In the same group, compared with the previous point in time: $\# \mathrm{P}<0.05$. 

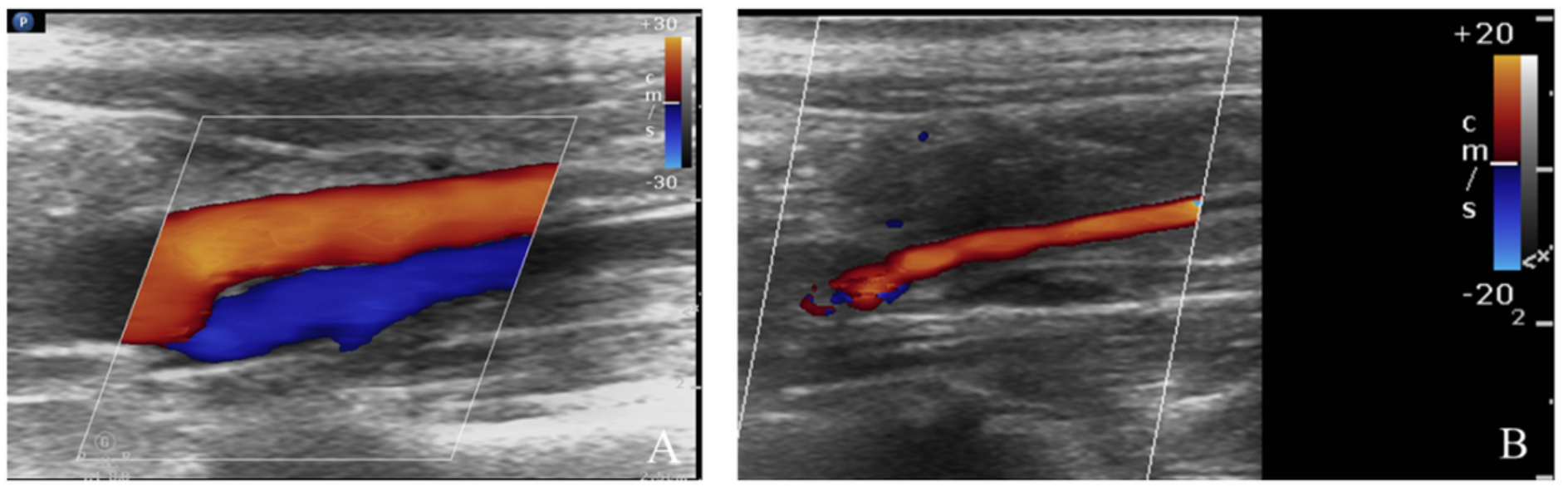

\section{Figure 6}

Color Doppler ultrasound was used to observe the changes in iliac artery blood flow before and after modeling. A: Before modeling, the internal diameter of the iliac artery was $0.31 \mathrm{~cm}$. Color Doppler ultrasound showed the blood flow of the iliac artery and iliac vein. B: After free bleeding for the 30s, color Doppler ultrasound showed that the artery rapidly collapsed with less blood flow and the inner diameter of the artery narrowed. The internal diameter of the iliac artery was $0.20 \mathrm{~cm}$. And color Doppler ultrasound showed that the iliac vein was compressed and there was no blood flow signal.
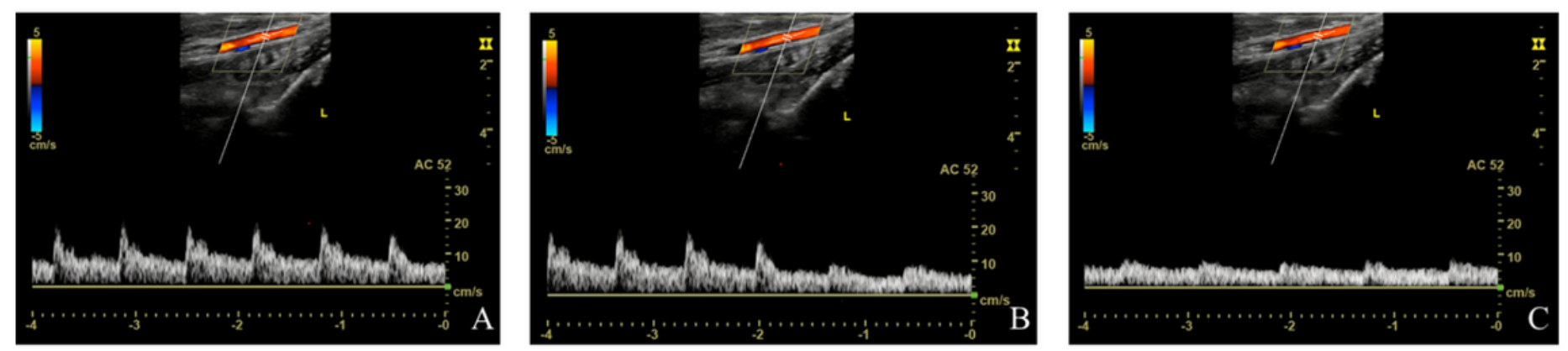

\section{Figure 7}

Once the balloon was inflated, the Doppler spectra confirmed a total occlusion of the arterial. A: It was the normal Doppler spectrum of the right iliac artery. B: With the balloon was inflating, the Doppler spectrum of the right iliac artery was changed. C: After the balloon inflated, the Doppler spectra confirmed a total occlusion of the arterial. 

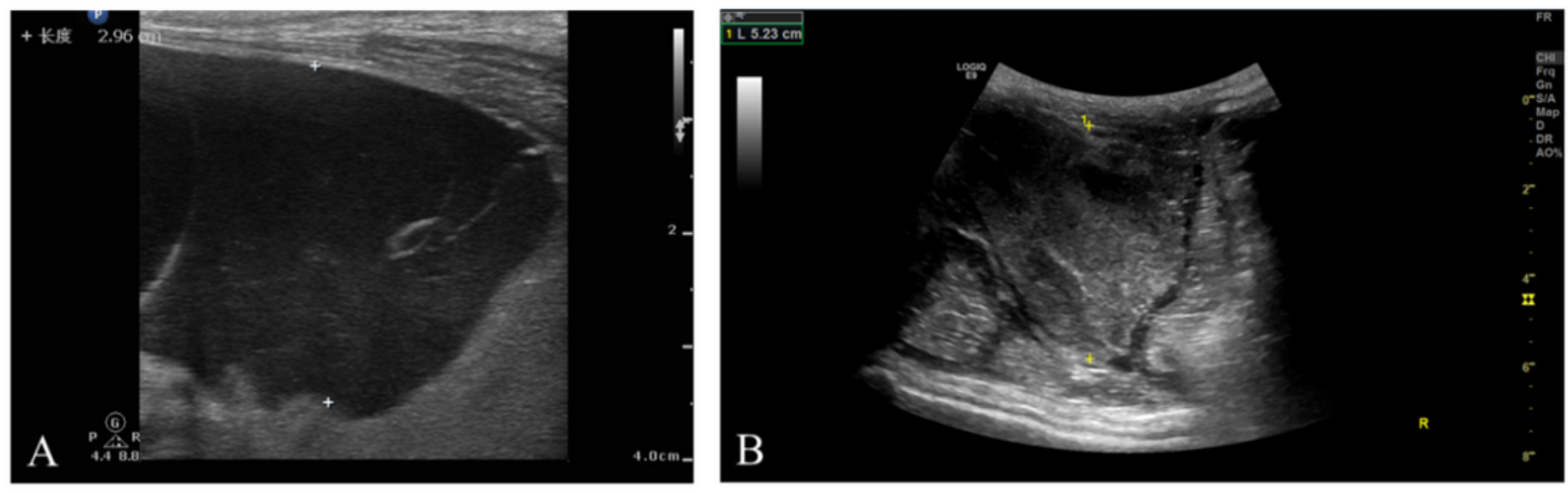

\section{Figure 8}

Comparison of the maximum depth of the anechoic zone in the abdominal cavity between the two groups at T4. A: The maximum depth of the anechoic zone in the abdominal cavity was $2.96 \mathrm{~cm}$, which was measured by portable ultrasound. B: In the control group, the maximum depth of the anechoic zone in the abdominal cavity was $5.23 \mathrm{~cm}$.
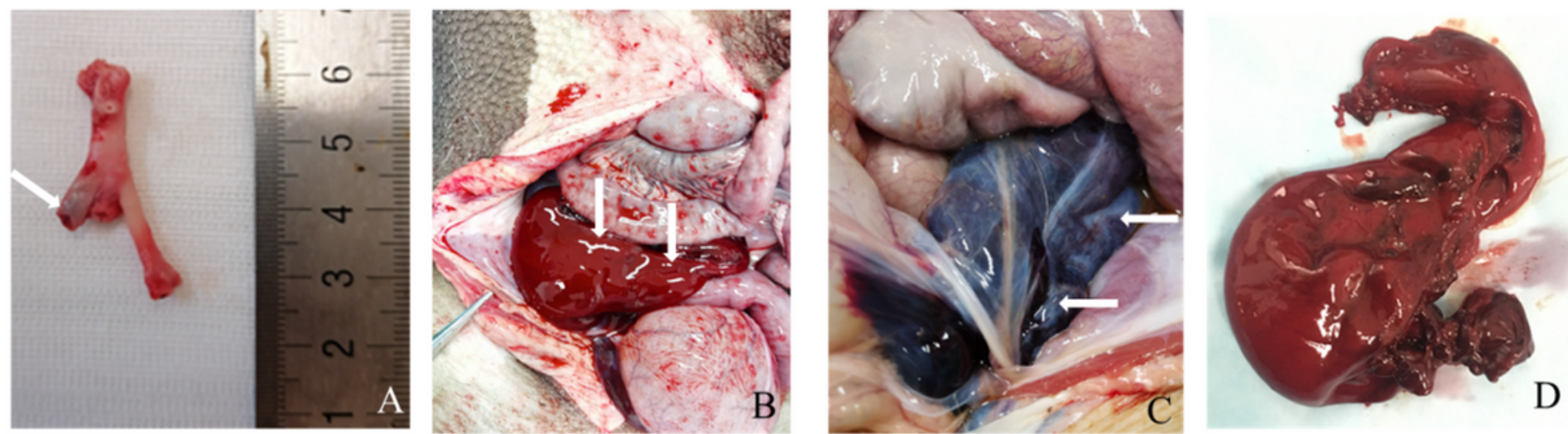

\section{Figure 9}

The gross specimen of the common iliac artery and huge blood clots. A: The gross specimen of the abdominal aorta and bilateral iliac artery. The arrow points to the rupture of the right iliac artery. B: After the animals were sacrificed and the abdominal was opened, huge blood clots in the abdominal cavity were visible. C: Huge blood clots in the retroperitoneum were visible. D: One of the abdominal cavity blood clots.
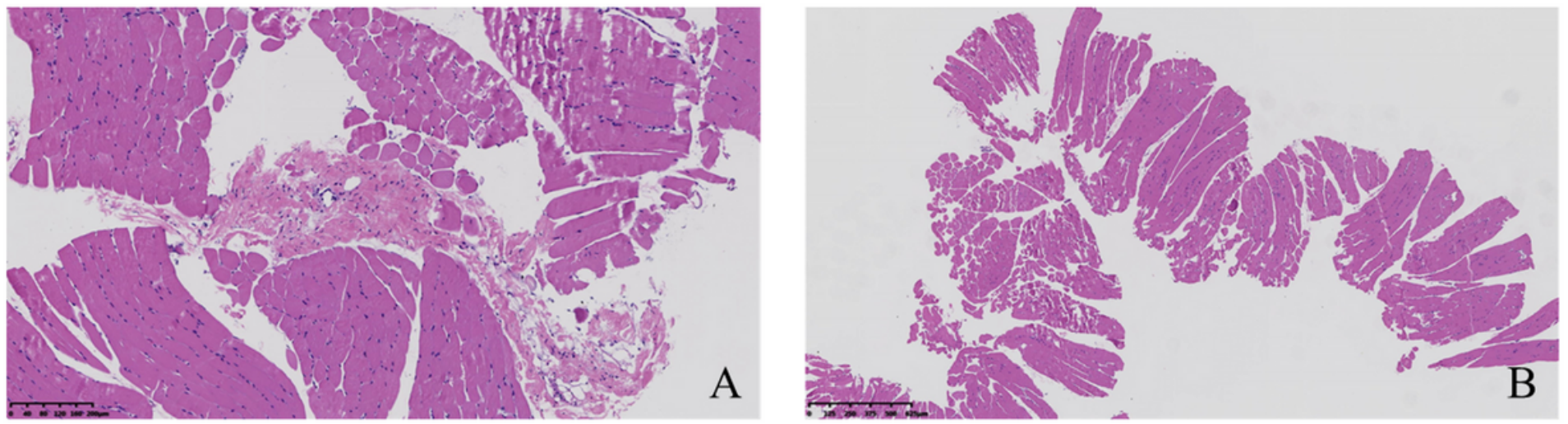
The muscle sections were stained with $\mathrm{HE}$ and the pathological changes of muscle tissue were observed under the light microscope in T1 and T4. A: The histopathological image of the right lower limb muscle in T1(10x). B: In the intervention group, no significant changes were observed in the same part of the right lower limb in T4 (4x).

\section{Supplementary Files}

This is a list of supplementary files associated with this preprint. Click to download.

- video11.mp4

- video2.mp4

- video3.mp4 